\title{
The possibilities of simultaneous detection of gamma rays, cosmic-ray electrons and positrons on the GAMMA-400 space observatory
}

\author{
A. M. Galper ${ }^{1,2}$, R. L. Aptekar ${ }^{3}$, I. V. Arkhangelskaya ${ }^{2}$, M. Boezio ${ }^{4}$, V. Bonvicini ${ }^{4}$, B. A. Dolgoshein ${ }^{2}$, M. O. Farber ${ }^{2}$, \\ M. I. Fradkin ${ }^{1}$, V. Ya. Gecha ${ }^{5}$, V. A. Kachanov ${ }^{6}$, V. A. Kaplin ${ }^{2}$, E. P. Mazets ${ }^{3}$, A. L. Menshenin ${ }^{5}$, P. Picozza ${ }^{7}$, \\ O. F. Prilutskii ${ }^{8}$, V. G. Rodin $^{8}$, M. F. Runtso ${ }^{2}$, P. Spillantini ${ }^{9}$, S. I. Suchkov ${ }^{1}$, N. P. Topchiev ${ }^{1}$, A. Vacchi ${ }^{4}$, \\ Yu. T. Yurkin ${ }^{2}$, N. Zampa ${ }^{4}$, and V. G. Zverev ${ }^{2}$ \\ ${ }^{1}$ Lebedev Physical Institute, Russian Academy of Sciences, Moscow, Russia \\ ${ }^{2}$ National Research Nuclear University "MEPhI”, Moscow, Russia \\ ${ }^{3}$ Ioffe Physical Technical Institute, Russian Academy of Sciences, St. Petersburg, Russia \\ ${ }^{4}$ Istituto Nazionale di Fisica Nucleare, Sezione di Trieste, Italy \\ ${ }^{5}$ All-Russia Research Institute of Electromechanics and Iosifyan Plant, Moscow, Russia \\ ${ }^{6}$ Institute for High Energy Physics, Protvino, Russia \\ ${ }^{7}$ Istituto Nazionale di Fisica Nucleare, Sezione di Roma 2 and Physics Dep. of University of Rome "Tor Vergata”, Rome, Italy \\ ${ }^{8}$ Space Research Institute, Russian Academy of Sciences, Moscow, Russia \\ ${ }^{9}$ Istituto Nazionale di Fisica Nucleare, Sezione di Firenze and Physics Department of University of Florence, Florence, Italy
}

Received: 29 October 2010 - Revised: 27 December 2010 - Accepted: 4 January 2011 - Published: 21 February 2011

\begin{abstract}
The GAMMA-400 space observatory will provide precise measurements of gamma rays, electrons, and positrons in the energy range $0.1-3000 \mathrm{GeV}$. The good angular and energy resolutions, as well as identification capabilities (angular resolution $\sim 0.01^{\circ}$, energy resolution $\sim 1 \%$, and proton rejection factor $\sim 10^{6}$ ) will allow us to study the main galactic and extragalactic sources, diffuse gamma-ray background, gamma-ray bursts, and to measure electron and positron fluxes. The peculiar characteristics of the experiment is simultaneous detection of gamma rays and cosmicray electrons and positrons, which can be connected with annihilation or decay of dark matter particles.
\end{abstract}

\section{Introduction}

The GAMMA-400, new gamma-ray space observatory, is developed by the GAMMA-400 international collaboration and contains two instruments: the GAMMA-400 gammaray telescope (Dogiel et al., 1988; Ginzburg et al., 2007,

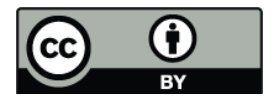

Correspondence to: S. Suchkov

(soutch@mail.ru) 2009a,b) and the KONUS-FG gamma-ray burst monitor (Apektar et al., 2009). The GAMMA-400 telescope will detect gamma rays, electrons, and positrons in the energy range from $100 \mathrm{MeV}$ up to $3000 \mathrm{GeV}$ with the angular and energy resolutions considerably better than in the earlier instruments EGRET (Thompson et al., 1993), AGILE (Tavani et al., 2009), Fermi-LAT (Atwood et al., 2009), PAMELA (Adriani et al., 2009), future AMS-2 (Beischer et al., 2009) and CALET (Torii et al., 2008). The scientific goals of GAMMA-400 originate from the results obtained by FermiLAT and other astrophysical space missions EGRET, AGILE, PAMELA, as well as the results from TeV ground-based gamma-ray facilities.

\section{Scientific problems}

\subsection{Investigation of high-energy gamma rays}

At present, the classical investigation of high-energy gamma rays cover a wide range of problems: understanding the mechanisms of charged particle acceleration in active galactic nuclei, pulsars, and supernova remnants, determining the nature of the still-unidentified Fermi-LAT sources, detailed

Published by Copernicus Publications on behalf of the Arbeitsgemeinschaft Extraterrestrische Forschung e.V. 


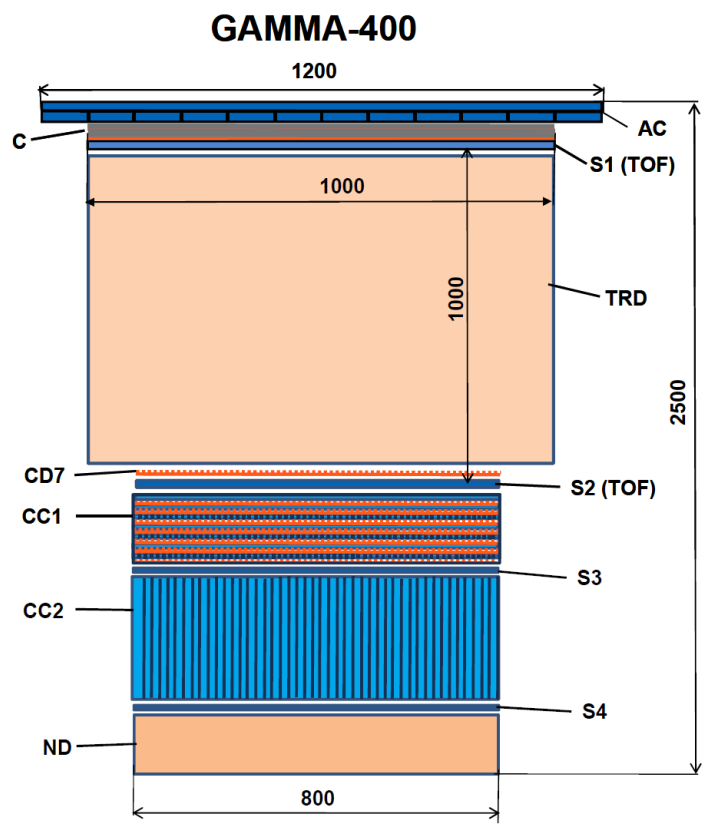

Fig. 1. The GAMMA-400 physical scheme.

studying the gamma-ray diffuse emission (both galactic, and extragalactic, as well as from molecular clouds), highenergy emission from gamma-ray bursts, transient gammaray sources, searching for the dark matter.

\subsection{The nature of dark matter and the sources of high- energy electrons and positrons}

According to some existing theoretical models, the dark matter particle annihilation or decay processes lead to generation of different elementary particles, including gamma rays, electrons, and positrons (Ullio et al., 1998). To detect them it is necessary to measure the energy spectra of annihilation or decay products, namely, the spectra of gamma rays and electron-positron component in the energy range 10$1000 \mathrm{GeV}$ with very good proton rejection.

The results of high-energy electron-positron component measurements will allow one to determine their generation sources, which could be known astrophysical objects as supernova remnants, pulsars, and others. In this case, there is the possibility to measure the spatial distribution of pulsars, to reveal physical conditions of electron-positron pair generation in large magnetic fields, particle escaping from the source into interstellar space, and conditions of their propagation in the interstellar matter.

However, the most important result of studying the highenergy electron-positron component would be the discovery of the correlation of these fluxes with the processes of annihilation or decay (or both processes simultaneously) of supersymmetrical weak interacting massive particles (WIMPs) such as neutralino, Kaluza-Klein bosons, and others. At the same time, there is the possibility to estimate WIMPs mass in the energy range inaccessible to particle accelerators.

\subsection{Requirements for new gamma-ray telescopes}

From the Fermi-LAT results in the energy range $100 \mathrm{MeV}-$ $100 \mathrm{GeV}$ we note the following:

- it is confirmed that many of the discrete sources in the gamma-ray range are variable;

- the number of the discovered discrete gamma-ray sources in comparison with the EGRET and AGILE data has increased by several times and has reached approximately 1500 , but considerable part of these sources remains unidentified;

- gamma-ray energy spectra from observations of FermiLAT near $100 \mathrm{GeV}$ and ground-based gamma-ray telescopes in the hundred $\mathrm{GeV}$ energy range have some discrepancies.

To explain many new problems occurred after the EGRET, AGILE, Fermi observations it is necessary to:

1. Extend the energy range up to several $\mathrm{TeV}$ (to better match space-based and ground-based observation data).

2. Improve angular resolution up to $\sim 0.01^{\circ}$ (to identify discrete sources).

3. Improve energy resolution up to $\sim 1 \%$ (to reveal features in the energy spectra of gamma rays, electrons, and positrons, which are found to be connected with the dark matter).

4. Increase the efficiency of gamma-ray selection.

\section{The GAMMA-400 description}

\subsection{Physical scheme}

GAMMA-400 is a pair-production telescope and its detection mode for gamma rays (after conversion) and electrons (positrons) is very similar, except for double energy released in detectors and anticoincidence detectors, in which there is a signal from primary electrons (positrons) and there is no signal for gamma rays. The GAMMA-400 physical scheme is shown in Fig. 1.

\subsection{Main systems of the gamma-ray telescope:}

- Anticoincidence detector (AC).

- Converter (C), multilayer converter, consists of 6 layers of 0.14 r.l. tungsten interleaved with $\mathrm{Si}(\mathrm{x}, \mathrm{y})$ strip coordinate detectors (CD1-CD6) with pitch $0.1 \mathrm{~mm}$. The total converter thickness is 0.84 r.l. 
- Time of flight system (TOF) consists of two scintillator detectors $\mathrm{S} 1$ and $\mathrm{S} 2$ at $1 \mathrm{~m}$ distance and is used to identify particles direction.

- Transition radiation detector (TRD) is used to improve proton rejection by a factor of $20-50$.

- Coordinate detector CD7 Si (x, y) strip coordinate detector with pitch $0.1 \mathrm{~mm}$

- Coordinate calorimeter (CC) consist of two parts: - imaging calorimeter (CC1): 10 layers of $1 \mathrm{~cm}$ BGO crystals and $\mathrm{Si}(\mathrm{x}, \mathrm{y})$ strip coordinate detectors with pitch $0.5 \mathrm{~mm}$. Total thickness is $\sim 9$ r.l.;

- electromagnetic calorimeter (CC2) made with BGO crystals. Total thickness is $\sim 21$ r.l.

- Scintillation detectors S3 and S4 are additional trigger detectors.

- Neutron detector (ND) improves proton rejection up to 10 times.

\subsection{Main features of the GAMMA-400 scheme}

To increase the angular resolution up to $\sim 0.01^{\circ}$ we use:

- multilayer converter, which allows us to precisely determine the conversion point and to measure particle energy up to $\sim 100 \mathrm{MeV}$;

- imaging calorimeter $\mathrm{CC} 1$, which allows us to precisely determine the shower axis;

large distance between converter and calorimeter $(\sim 1 \mathrm{~m})$.

To extend the energy range up to $3000 \mathrm{GeV}$ we use thick calorimeter $(\sim 30$ r.l.) $\quad$ consisting of two parts (imaging calorimeter and electromagnetic calorimeter).

To increase the energy resolution up to $1 \%$ we use:

- imaging calorimeter from BGO crystals interleaved with Si strip detectors with pitch $0.5 \mathrm{~mm}$;

- electromagnetic calorimeter made with BGO crystals.

To provide proton rejection factor up to $10^{6}$ we use the shower shape in the calorimeter, as well as the data from the transition radiation detector and the neutron detector.

To prevent self-veto of the telescope by backsplash effect, we use a method based on measuring the time instant of signals in AC and S1 for backsplash particles in contrast to primary gamma rays allowing us to decrease considerably the backsplash effect.

The GAMMA-400 expected performances obtained by simulations using the Monte-Carlo method are presented in Table 1.
Table 1. The GAMMA-400 expected performances.

\begin{tabular}{ll}
\hline \multicolumn{2}{c}{ GAMMA-400 gamma-ray telescope } \\
Gamma-ray energy range & $0.1-3000 \mathrm{GeV}$ \\
Converter (area and thickness) & $100 \times 100 \mathrm{~cm}^{2}, 0.84 \mathrm{r} .1$. \\
Calorimeter (area and thickness) & $80 \times 80 \mathrm{~cm}^{2} \sim 30 \mathrm{r} .1$. \\
Field of view & $\pm 50^{\circ}$ \\
Angular resolution $\left(E_{\gamma}>100 \mathrm{GeV}\right)$ & $\sim 0.01^{\circ}$ \\
Energy resolution $\left(E_{\gamma}>10 \mathrm{GeV}\right)$ & $\sim 1 \%$ \\
Proton rejection factor & $10^{6}$ \\
Point source sensitivity, ph/cm ${ }^{2} \mathrm{~s}$ & $\sim 2 \times 10^{-9}$ \\
$\left(E_{\gamma}>100 \mathrm{MeV}\right)$ & $100 \mathrm{~GB} / \mathrm{day}$ \\
Telemetry downlink & $2000 \mathrm{~W}$ \\
Power consumption & $2 \times 2 \times 3.0 \mathrm{~m}^{3}$ \\
Maximum dimensions & $\sim 2600 \mathrm{~kg}$ \\
Total mass & $>7$ years \\
Lifetime KONUS-FG gamma-ray burst monitor \\
\hline \multicolumn{1}{c}{$10 \mathrm{keV}-10 \mathrm{MeV}$} \\
Energy range
\end{tabular}

\section{Observation conditions}

The Gamma-400 space observatory will be installed on the Navigator space service platform produced by Lavochkin Research and Production Association. GAMMA-400 will be launched into elliptical orbit with initial parameters: apogee $300000 \mathrm{~km}$, perigee $500 \mathrm{~km}$, inclination $51.8^{\circ}$. The orbit period is 7 days. The spacecraft lifetime is more than 7 years. We expect to use three basic modes of observation: mode of gamma-ray sky monitoring (searching for new discrete sources and monitoring the known variable sources); long-term monitoring of the most interesting discrete sources; quick observatory reorientation according to signal from KONUS-FG to observe $\mathrm{GeV}$ gamma-ray burst with the GAMMA-400 telescope and according to the command from the Earth about extraordinary information from other astronomical observations, like, for example, information on a solar flare.

The launch of the GAMMA-400 space observatory is planned in 2017

Acknowledgements. This work was supported by the Russian Space Agency and Space Council of the Russian Academy of Sciences.

Edited by: R. Vainio

Reviewed by: D. J. Thompson and another anonymous referee 


\section{References}

Adriani, O., Barbarino, G. C., Bazilevskaya, G. A., et al.: An anomalous positron abundance in cosmic rays with energies $1.5-$ $100 \mathrm{GeV}$, Nature, 458, 607-609, 2009.

Aptekar, R. L., Cline, T. L., Frederiks, D. D., et al.: KONUSWIND observations of the new soft gamma-ray repeater SGR 0501+4516, Astroph. J., 698, L82-L85, 2009.

Atwood, W. B. for the Fermi/LAT Collaboration: The large area telescope on the FERMI gamma-ray space telescope mission, Astroph. J., 697, 1071-1102, 2009.

Beischer, B., von Doetinchem, P., Gast, H., Kirn, T., and Schael, S.: Perspectives for indirect dark matter search with AMS-2 using cosmic-ray electron and positron, New J. of Physics, 11, 105021-105035, 2009.

Dogiel, V. A., Fradkin, M. I., Kurnosova, L. V., et al.: Some tasks of observational gamma-ray astronomy in the energy range 5400 GeV: Space Science Rev., 49, 215-226, 1988.

Ginzburg, V., Kaplin, V., Karakash, A., et al.: Development of the GAMMA-400 gamma-ray telescope to record cosmic gamma rays with energies up to $1 \mathrm{TeV}$, Kosm. Issled., 45, 449-451, 2007.
Ginzburg, V., Kaplin, V., Rutco, M., et al.: Advanced GAMMA-400 gamma-ray telescope for recording cosmic gamma rays with energies up to $3 \mathrm{TeV}$, Bulletin of the Russian Academy of Sciences: Physics, 73, 664-666, 2009a.

Ginzburg V., Galper, A., Fradkin, M., et al.: The GAMMA-400 project, Preprint LPI, no. 10, $2009 \mathrm{~b}$.

Tavani, M. for the AGILE Collaboration: The AGILE mission, Astron. Astrophys., 502, 995-1013, 2009.

Thompson, D. J., Bertsch, D. L., Fichtel, C. E., et al.: Calibration of the energetic gamma-ray experiment telescope (EGRET) for the Compton gamma-ray observatory, Astroph. J., 86, 629-656, 1993.

Torii, S. for the CALET Collaboration: The Calorimetric Electron Telescope (CALET) Project, J. Phys. Soc. Jpn., 77, 41-44, 2008.

Ullio, P., Buckley, J. H., and Bergström, L.: Observability of rays from dark matter neutralino annihilations in the Milky Way halo, Astroparticle Physics, 9, 137-162, 1998. 